\title{
Role of actin cytoskeleton in mammalian sperm capacitation and the acrosome reaction
}

\author{
Haim Breitbart, Gili Cohen and Sara Rubinstein \\ Faculty of Life Sciences, Bar-Ilan University, Ramat-Gan 52900, Israel \\ Correspondence should be addressed to H Breitbart; Email: breith@mail.biu.ac.il
}

\begin{abstract}
In order to fertilize, the mammalian spermatozoa should reside in the female reproductive tract for several hours, during which they undergo a series of biochemical modifications collectively called capacitation. Only capacitated sperm can undergo the acrosome reaction after binding to the egg zona pellucida, a process which enables sperm to penetrate into the egg and fertilize it. Polymerization of globular (G)-actin to filamentous (F)-actin occurs during capacitation, depending on protein kinase A activation, protein tyrosine phosphorylation, and phospholipase $\mathrm{D}$ activation. F-actin formation is important for the translocation of phospholipase $C$ from the cytosol to the sperm plasma membrane during capacitation. Prior to the occurrence of the acrosome reaction, the F-actin should undergo depolymerization, a necessary process which enables the outer acrosomal membrane and the overlying plasma membrane to come into close proximity and fuse. The binding of the capacitated sperm to the zona pellucida induces a fast increase in sperm intracellular calcium, activation of actin severing proteins which break down the actin fibers, and allows the acrosome reaction to take place.

Reproduction (2005) 129 263-268
\end{abstract}

\section{Introduction}

In mammalian species, sperm-egg interaction and mutual activation are mediated by the zona pellucida (ZP), the glycoprotein coat of the egg (reviewed in Wassarman 1988). The spermatozoon binds to the ZP with its plasma membrane intact, via specific receptors that are localized over the anterior head region of the sperm. The binding of the sperm to the ZP stimulates it to undergo an acrosome reaction, which enables the sperm to penetrate and fertilize the egg (reviewed in Yanagimachi 1994).

The binding of the sperm to the egg and the occurrence of the acrosome reaction will take place only if the sperm has previously undergone a poorly defined maturation process in the female reproductive tract, known as capacitation. We recently reviewed the known signal transduction events occurring during capacitation and the acrosome reaction (Breitbart 2003). The possible changes and regulation of the sperm actin cytoskeleton as part of the mechanisms of capacitation and the acrosome reaction are described in this review.

\section{Actin and related proteins in sperm}

In spermatogenic cells, actin filaments have been described primarily in the subacrosomal space between the nucleus and the developing acrosome of spermatids of certain mammalian species (Vogl 1989). In mature spermatozoa, however, the structure and location of actin filaments have not been made clear. In most reports, actin seems to be present in its monomeric form, although filamentous (F)-actin has been described in mammalian species as well (Flaherty 1987, Breed \& Leigh 1991, Moreno-Fierros et al. 1992, Vogl et al. 1993, de las Heras et al. 1997, Howes et al. 2001). In human sperm the regions reported to contain actin include the acrosomal space, the equatorial and post acrosomal regions, and the tail (Clarke et al. 1982, Virtanen et al. 1984, Ochs \& Wolf 1985, Fouquet \& Kann 1992). The presence of actin in the tail might be important for the regulation of sperm motility, and its presence in the head suggests a possible involvement in the acrosome reaction. It was reported that actin polymerization is important for initiation of sperm motility during posttesticular maturation (Lin et al. 2002). The location of actin in the acrosomal region of several mammalian species including hamster, boar, human, bull, rabbit and guinea-pig (Talbot \& Kleve 1978, Camatini et al. 1986, Flaherty et al. 1988, Olson \& Winfrey 1991, MorenoFierros et al. 1992, Yagi \& Paranko 1995) supports its possible role in sperm capacitation and the acrosome reaction. Actin polymerization is necessary for sperm incorporation into the egg cytoplasm (Sanchez-Gutierrez et al. 2002) and for sperm nuclei decondensation 
(Kumakiri et al. 2003). The assembly of G-actin to form F-actin is controlled by several actin-binding proteins. The existence of proteins such as calicin (von Bulow et al. 1995), the capping proteins CPß3 (von Bulow et al. 1997) and CP $\alpha 3$ (Tanaka et al. 1994), destrin, thymosin $\beta 10$, testis-specific actin capping protein (Howes et al. 2001), gelsolin (de las Heras et al. 1997), scinderin (Pelletier et al. 1999), and the actin-related proteins Arp-T1 and T2 (Heid et al. 2002) in mammalian sperm suggests that actin polymerization and depolymerization might be involved in sperm function. In boar and guinea-pig sperm, actin polymerization occurs during capacitation (Castellani-Ceresa et al. 1993, Cabello-Agueros et al. 2003). Recently, we showed that actin polymerization occurs during capacitation of bull, mouse, human, and ram sperm, whereas F-actin breakdown should occur in order to achieve the acrosome reaction (Brener et al. 2003). In human sperm, actin is lost from the acrosomal region following the acrosome reaction (AR) (Liu et al. 1999) and blocking actin polymerization inhibited ZPinduced AR (Liu et al. 1999, 2002). In addition, inhibition of actin breakdown blocks bovine sperm AR (Spungin et al. 1995). Moreover, inhibition of actin polymerization in guinea-pig and human sperm by cytochalosin D, blocks sperm penetration into zona-free hamster eggs (Rogers et al. 1989) as well as the in vitro fertilization ability of boar (Castellani-Ceresa et al. 1993) and mouse (Brener et al. 2003) sperm. These evidences suggest that remodeling of actin structure plays an important role in sperm capacitation and the AR.

\section{Protein tyrosine phosphorylation and actin polymerization}

It is accepted that protein kinase A (PKA)-dependent tyrosine phosphorylation of several proteins occurs during sperm capacitation (Visconti et al. 1995). In human and bovine sperm, reactive oxygen species (ROS) up-regulates protein tyrosine phosphorylation (Aitken et al. 1995, Leclerc et al. 1997, Rivlin et al. 2004), consistent with the suggestion that hydrogen peroxide activates adenylyl cyclase to produce cAMP which activates PKA (Aitken 1997, Rivlin et al. 2004).

It is unclear whether a specific ligand induces the signal transduction cascade leading to protein tyrosine phosphorylation in sperm capacitation. One possible ligand is the epidermal growth factor (EGF), which interacts with its receptor (EGFR) identified in the bovine sperm head (Lax et al. 1994). EGF stimulates the tyrosine phosphorylation of several sperm proteins (Breitbart et al. 1995) and activates phospholipase $\mathrm{C}_{\gamma}(\mathrm{PLC} \gamma)$ (Spungin et al. 1995) and actin polymerization (Brener et al. 2003, Cohen et al. 2004) in bovine sperm capacitation.

There is a good correlation between actin polymerization and protein tyrosine phosphorylation in bovine and ram sperm capacitation (Brener et al. 2003). In bovine sperm, the two processes do not occur in the absence of bicarbonate; both depend on PKA and tyrosine kinase activities, both are enhanced by EGF, hydrogen peroxide, and the tyrosine phosphatase inhibitor vanadate, and both are blocked by glucose (Brener et al. 2003). These data suggest that protein tyrosine phosphorylation and actin polymerization are related processes occurring in sperm capacitation. Interestingly, we found that EGF, hydrogen peroxide, or vanadate in the absence of bovine serum albumin (BSA) in the incubation medium, cannot, by themselves, induce capacitation (measured by percent of acrosome reacted cells), although they stimulate tyrosine phosphorylation and actin polymerization (Brener et al. 2003). This indicates that these two processes are necessary but insufficient for achieving sperm capacitation. The role of BSA is to increase cholesterol efflux from the plasma membrane, which should occur in order to capacitate the sperm.

We show elsewhere (Cohen et al. 2004) and here (Table 1 ) that actin polymerization in bovine sperm is

Table 1 The effect of various inhibitors on actin polymerization of bovine sperm following incubation with various activators. The numbers represent relative fluorescence intensity \pm S.E.M. The fluorescence at zero time (before starting the incubation) was $24 \pm 1$. Percent inhibition was calculated after subtracting the zero time fluorescence. The various inhibitors were used at concentrations in which they represent specific inhibition.

\begin{tabular}{|c|c|c|c|c|c|c|c|c|}
\hline \multirow[b]{2}{*}{ Inhibitor } & \multirow[b]{2}{*}{ No activator (4 h) } & \multicolumn{7}{|c|}{ Activator } \\
\hline & & $\%$ Inhibition & PMA (30 min) & $\%$ Inhibition & dbcAMP (10 min) & $\%$ Inhibition & $\mathrm{PA}(3 \mathrm{~min})$ & $\%$ Inhibition \\
\hline None & $100 \pm 3$ & - & $102 \pm 3$ & - & $92 \pm 2$ & - & $112 \pm 2$ & - \\
\hline BAРТA-AM & $26 \pm 2$ & 97 & $25 \pm 1$ & 99 & $88 \pm 1$ & 6 & $100 \pm 3$ & 14 \\
\hline Genestein & $32 \pm 1$ & 90 & $39 \pm 1$ & 81 & $45 \pm 1$ & 69 & $83 \pm 3$ & 33 \\
\hline PD98059 & $30 \pm 1$ & 92 & $28 \pm 1$ & 95 & $30 \pm 2$ & 91 & $89 \pm 2$ & 26 \\
\hline Brefeldin & $28 \pm 2$ & 95 & $31 \pm 2$ & 91 & $30 \pm 1$ & 91 & $90 \pm 2$ & 25 \\
\hline Neomycin & $24 \pm 1$ & 100 & $24 \pm 2$ & 100 & $31 \pm 1$ & 90 & $90 \pm 2$ & 25 \\
\hline
\end{tabular}

Bovine sperm were incubated for the indicated times in the presence of the PKC stimulator phorbol myristyl acetate (PMA, 100 ng/ml), PKA activator dibutyryl cAMP (dbcAMP, $1 \mathrm{mM})$ or the PLD product phosphatidic acid (PA) $(3 \mu \mathrm{g} / \mathrm{ml})$. Before this incubation, the cells were incubated for $1 \mathrm{~h}$ with the $\mathrm{Ca}^{2+}$ chelator BAPTA-AM or for $10 \mathrm{~min}$ with the tyrosine kinase inhibitor genestein $(20 \mu \mathrm{g} / \mathrm{ml})$, the MAPKK inhibitor PD98059 $(25 \mu \mathrm{M})$, the ARF inhibitor brefeldin $(10 \mu \mathrm{M})$ or the PLC inhibitor neomycin $(5 \mathrm{mM})$. At the end of incubation, samples were stained with FITC-phalloidin as described by us elsewhere (Cohen et al. 2004) and the fluorescence intensity in the sperm head was determined quantitatively using the Meta Morph Image $\mathrm{J}$ and Adobe Photoshop processing software. 
significantly enhanced after short incubation of the cells with dibutyryl-cAMP (dbcAMP) or the phorbol ester phorbol myristyl acetate (PMA), which activate PKA or protein kinase $\mathrm{C}(\mathrm{PKC})$ respectively. This F-actin formation is almost completely blocked by the tyrosine kinase inhibitor genestein (Table 1). This suggests that protein tyrosine phosphorylation is involved in PKA- and PKC-induced actin polymerization, although we could not observe any stimulation in tyrosine phosphorylated proteins under these conditions (Rivlin et al. 2004). It is possible that the determination of tyrosine phosphorylation using anti-phosphotyrosine is not sensitive enough to detect very small changes in phosphorylation. This point needs further clarification. The suggested activation of the tyrosine kinase is probably needed for the activation of phospholipase D (PLD) (see below), since actin polymerization induced by exogenous phosphatidic acid (PA) (the product of PLD activity) was only slightly inhibited by genestein (Table 1).

In our recent studies, we show that in bicarbonatedeficient medium, hydrogen peroxide could induce protein tyrosine phosphorylation and actin polymerization, two processes which are essential but not sufficient for capacitation (Brener et al. 2003, Rivlin et al. 2004). We also show that $\mathrm{H}_{2} \mathrm{O}_{2}$ stimulates sperm adenylyl cyclase (AC) and tyrosine phosphorylation of the $80 \mathrm{KDa}$ protein in addition to other proteins which are tyrosine-phosphorylated under regular capacitation conditions as well (Rivlin et al. 2004). The $\mathrm{H}_{2} \mathrm{O}_{2}$-stimulated phosphorylation of the $80 \mathrm{KDa}$ protein as well the phosphorylation of an $85 \mathrm{KDa}$ protein are both dependent on intracellular $\mathrm{Ca}^{2+}$ concentration $\left(\left[\mathrm{Ca}^{2+}\right]_{1}\right)$ (Rivlin et al. 2004). Actin polymerization occurred during capacitation (Table 1) or, induced by exogenous $\mathrm{H}_{2} \mathrm{O}_{2}$ (not shown), was almost completely blocked by chelating intracellular $\mathrm{Ca}^{2+}$. F-actin formation induced by dbcAMP was only slightly inhibited by chelating intracellular $\mathrm{Ca}^{2+}$ (Table 1), suggesting that AC activation depends upon $\left[\mathrm{Ca}^{2+}\right]_{\mathrm{i}}$.

Since tyrosine phosphorylation of the $80 \mathrm{KDa}$ protein and actin polymerization are $\left[\mathrm{Ca}^{2+}\right]_{i}$-dependent processes but not in dbcAMP-treated cells, we suggest that $\mathrm{H}_{2} \mathrm{O}_{2}$ activates a $\mathrm{Ca}^{2+}$-dependent tyrosine kinase in addition to its direct effect on sperm AC. We must emphasize that the bicarbonate-dependent tyrosine phosphorylation of 8 different sperm proteins is not dependent on $\left[\mathrm{Ca}^{2+}\right]_{i}$ (Rivlin et al. 2004). Thus, the sperm soluble AC, known to be activated by bicarbonate (Chen et al. 2000), is relatively insensitive to $\left[\mathrm{Ca}^{2+}\right]_{\mathrm{i}}$, whereas the tyrosine phosphorylation of the $80 \mathrm{KDa}$ and $85 \mathrm{KDa}$ proteins as well as actin polymerization are $\left[\mathrm{Ca}^{2+}\right]_{i-}$ dependent processes. This may suggest that actin polymerization depends on $\mathrm{Ca}^{2+}$-dependent tyrosine kinase(s) which leads to the tyrosine phosphorylation of $80 \mathrm{KDa}$ and $85 \mathrm{KDa}$ sperm proteins.

\section{Actin polymerization is regulated by phospholipase D (PLD)}

In a recent study, we showed that PLD is involved in bovine sperm actin polymerization during capacitation (Cohen et al. 2004). We also demonstrated that the isoform PLD1 is localized mainly in the acrosomal region of bovine sperm (Garbi et al. 2000), suggesting its possible involvement in the acrosome reaction. The requirement of PLD activity for F-actin formation is based on the following: first, actin polymerization is significantly inhibited by the PLD inhibitors butan-1-ol and C2-ceramide but not by butan-2-ol; secondly, exogenous PLD or PA stimulates actin polymerization which is not affected by butan-1-ol, and finally, PLD activity is enhanced during capacitation prior to F-actin formation (Cohen et al. 2004).

Relatively fast (within 10-20 min) PLD activation and actin polymerization are induced by activating PKA or PKC, and these activities are completely blocked by butan-1-ol, indicating that PLD mediates these activities. Indeed, we show that PLD is activated in sperm by activation of PKA or PKC (Cohen et al. 2004). In bovine sperm, PKC $\alpha$ and PLD1 co-exist as a complex, which decomposes after PKC activation (Garbi et al. 2000). This complex is decomposed by activating PKC using its direct activator PMA or by activating the lysophosphatidic acid (LPA) receptor (Garbi et al. 2000) resulting in PLD activation (Cohen et al. 2004).

Inhibition of PKA activity throughout the four hours of sperm capacitation completely blocked actin polymerization, while inhibition of PKC revealed only partial (40\%) inhibition (Cohen et al. 2004). These findings are in agreement with the notion regarding the obligatory role of PKA in sperm capacitation (Visconti et al. 1995). However, we found that activation of sperm PKC induced fast (20-30 min) PKA-independent actin polymerization (Cohen et al. 2004). Moreover, when PKA was blocked (using $\mathrm{H}-89$ or bicarbonate-deficient medium), there was a rapid $(30 \mathrm{~min})$ increase in F-actin, which was inhibited by PKC inhibition, as well as PKC $\alpha$ activation (Cohen et al. 2004). The effect of H-89 or of bicarbonate-deficient medium on actin polymerization was blocked by inhibition of phospholipase C (PLC) activity, suggesting that PKA inhibits PLC activity in bovine sperm (Cohen et al. 2004). When PKA is blocked, PLC can be activated leading to PKC and PLD activation and actin polymerization. Neomycin binds to phosphtidilinositol-4,5 biphosphate $\left(\mathrm{PIP}_{2}\right)$ (the substrate of PLC and a cofactor for PLD) and inhibits the activity of these two enzymes as well as actin polymerization (Table 1). However, when actin polymerization is induced by PMA, conditions in which there is no need for PLC activity in order to activate PKC, actin polymerization is inhibited by neomycin (Table 1 ) but not by the PLC-specific inhibitor U73122 (Cohen et al. 2004), indicating that neomycin blocks PLD activation by its binding to $\mathrm{PIP}_{2}$. The fact that neomycin causes only a small inhibition in actin polymerization induced by exogenous PA 
(Table 1), supports this notion. PA is the final product of PLD activity, therefore endogenous PLD activity is not needed when exogenous PA is added to the cells. We also show here that inhibition of MAP kinase-kinase (MAPKK) or ADP-ribosylation factor (ARF) revealed high inhibition in actin polymerization induced under capacitation or by PMA or dbcAMP, but relatively low inhibition when exogenous PA is added to the cells (Table 1). This indicates that MAPK and ARF are involved in PLD activation. To summarize this point, we suggest that PLD can be activated by PKC or PKA pathways via MAPK and ARF activation leading to actin polymerization. In addition, PLD can be activated by cAMP independent of PKA, but this activation does not lead to actin polymerization (Cohen

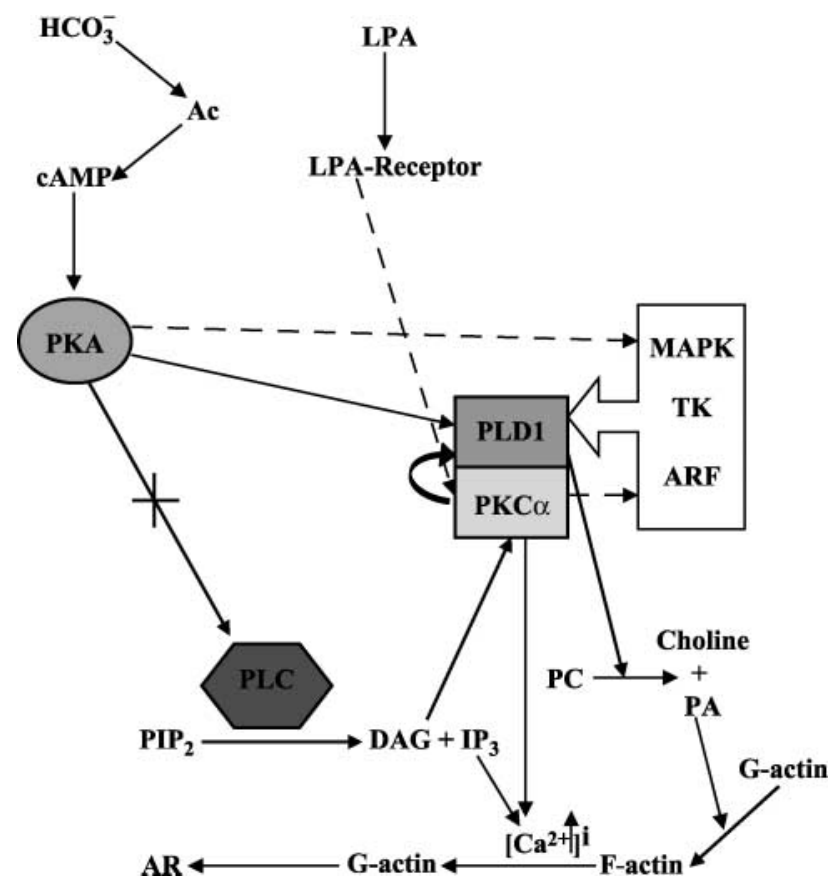

Figure 1 Remodeling of actin in sperm capacitation and the acrosome reaction (AR). G-actin is polymerized to F-actin during sperm capacitation and the fibers should undergo depolymerization in order to accomplish the AR. Actin polymerization depends on PLD activation, which occurs via the $\mathrm{HCO}_{3}^{-}$/CAMP/PKA pathway or via the G-protein coupled receptor (GPCR) (LPA-receptor)/PKC pathway. One of the GPCRs in sperm is LPA-receptor which can be activated by LPA, resulting in PKC activation (Garbi et al. 2000) and PLD-dependent actin polymerization (Cohen et al. 2004). MAP-kinase (MAPK), tyrosine kinase (TK), and ADP-ribosylation factor (ARF) are involved in PLD activation, leading to phosphatidyl-choline (PC) hydrolysis to produce phosphatidic acid (PA), which mediates polymerization of $\mathrm{G}$-actin to F-actin. The binding of capacitated sperm to the egg zona pellucida activates sperm PLC (Tomes et al. 1996) to hydrolyze $\mathrm{PIP}_{2}$ to diacylglycerol (DAG) and inositol triphosphate $\left(\mathrm{IP}_{3}\right)$. DAG further activates PKC, and $\mathrm{IP}_{3}$ activates the $\mathrm{Ca}^{2+}$ channel in the outer acrosomal membrane resulting in an increase in intracellular $\mathrm{Ca}^{2+}$ concentration $\left(\left[\mathrm{Ca}^{2+}\right]_{\mathrm{i}}\right)\left(\mathrm{O}^{\prime}\right.$ Toole et al. 2000). The high increase in $\left[\mathrm{Ca}^{2+}\right]_{\mathrm{i}}$, activates actin-severing proteins to break down $\mathrm{F}$-actin to $\mathrm{G}$-actin and accomplish the AR. et al. 2004). A model describing the various pathways is shown in Fig. 1.

\section{The crosstalk between PKA and PKC in sperm capacitation}

We previously described the gradual binding and activation of PLC $\gamma$ to the plasma membrane of bovine sperm during capacitation (Spungin \& Breitbart 1996). Since PKC activity mediates the acrosome reaction (Breitbart et al. 1992), we assume that the described activation of PLC $\gamma$ would result in PKC activation prior to the acrosome reaction. We showed that activation of PKC during bovine sperm capacitation causes a rapid increase in actin polymerization which is followed by fast depolymerization (Cohen et al. 2004), probably due to the increase in $\left[\mathrm{Ca}^{2+}\right]_{i}$ (Spungin \& Breitbart 1996, Brener et al. 2003). However, because high F-actin formation is needed at the end of the capacitation time in order to capacitate the sperm (Brener et al. 2003) and this cannot be reached when PKC is highly activated (Cohen et al. 2004), capacitation cannot be obtained. Thus, PKC activity should be kept low during sperm capacitation and this is accomplished by activation of PKA which blocks PLC/PKC activities (Cohen et al. 2004). Activation of PLC prior to the acrosome reaction would require downregulation of PKA towards the end of the capacitation time. This possibility is supported by others who have shown decreasing activity of adenyl cyclase towards the end of capacitation of mouse and human sperm (Adeoya-Osiguwa \& Fraser 2002, Lefievre et al. 2002).

It seems that under nonphysiological conditions, activation of PKA or PKC can independently cause PLD activation, leading to actin polymerization (Cohen et al. 2004). However, under physiological conditions, the PKA pathway is obligatory for actin polymerization and capacitation, whereas the PKC pathway is important for the acrosome reaction (see model in Fig. 1).

In summary, although PKA or PKC can lead to actin polymerization, a refined balance between the two pathways is required for optimal and sustained activation during sperm capacitation. The activation of PKA would cause inhibition of PLC, and prevent PKC activation during capacitation. It appears that PKA activation promotes capacitation whereas early activation of PKC jeopardizes capacitation. Thus, it would be necessary for inhibition of PKA activity to occur at the end of capacitation in order to achieve PKC activation prior to the acrosome reaction.

\section{Acknowledgements}

This research was supported by the Lhel Foundation to $\mathrm{HB}$. The authors declare that there is no conflict of interest that could prejudice the impartiality of this work. 


\section{References}

Adeoya-Osiguwa SA \& Fraser LR 2002 Capacitation state-dependent changes in adenosine receptors and their regulation of adenylyl cyclase/cAMP. Molecular Reproduction and Development 63 245-255.

Aitken RJ 1997 Molecular mechanisms regulating human sperm function. Molecular Human Reproduction 3 169-173.

Aitken RJ, Paterson M, Fisher H, Buckingham DW \& Van Duim M 1995 Redox regulation of tyrosine phosphorylation in human spermatozoa and its role in the control of human sperm function. Journal of Cell Science 108 2017-2025.

Breed WG \& Leigh CM 1991 Distribution of filamentous actin in and around spermatids and in spermatozoa of Australian conilurine rodents. Molecular Reproduction and Development $\mathbf{3 0}$ 369-384.

Breitbart H 2003 Signaling pathways in sperm capacitation and acrosome reaction. Cellular and Molecular Biology 49 $321-327$.

Breitbart H, Lax Y, Rotem R \& Naor Z 1992 Role of protein kinase C in the acrosome reaction of mammalian spermatozoa. Biochemical Journal $281473-476$

Breitbart H, Rubinstein S, Spungin B \& Lax Y 1995 Phosphorylation and cellular localization of signal transduction elements involved in sperm capacitation and acrosome reaction. Biology of Reproduction 52 (Suppl 1) 61.

Brener E, Rubinstein S, Cohen G, Shternall K, Rivlin J \& Breitbart H 2003 Remodeling of the actin cytoskeleton during mammalian sperm capacitation and acrosome reaction. Biology of Reproduction 68 837-845.

von Bulow M, Heid H, Hess H \& Franke WW 1995 Molecular nature of calicin, a major basic protein of the mammalian sperm head cytoskeleton. Experimental Cell Research 219 407-413.

von Bulow M, Rackwitz HR, Zimbelmann R \& Franke WW 1997 CP beta3, a novel isoform of an actin-binding protein, is a component of the cytoskeletal calyx of the mammalian sperm head. Experimental Cell Research 233 216-224.

Cabello-Agueros JF, Hernandez-Gonzalez EO \& Mujica A 2003 The role of F-actin cytoskeleton-associated gelsolin in the guinea pig capacitation and acrosome reaction. Cell Motility and the Cytoskeleton 56 94-108.

Camatini M, Anelli G \& Casale A 1986 Identification of actin in boar spermatids and spermatozoa by immunoelectron microscopy. European Journal of Cell Biology 42 311-318.

Castellani-Ceresa L, Mattioli M, Radaelli G, Barboni B \& Brivio MF 1993 Actin polymerization in boar spermatozoa: fertilization is reduced with use of cytochalasin D. Molecular Reproduction and Development 36 203-211.

Chen Y, Cann MJ, Litvin TN, lourgenko V, Sinclair ML, Levin LR \& Buck J 2000 Soluble adenylyl cyclase as an evolutionarily conserved bicarbonate sensor. Science 289 625-628.

Clarke GN, Clarke FM \& Wilson S 1982 Actin in human spermatozoa. Biology of Reproduction 26 319-327.

Cohen G, Rubinstein S, Gur Y \& Breitbart H 2004 Crosstalk between protein kinase $\mathrm{A}$ and $\mathrm{C}$ regulates phospholipase $\mathrm{D}$ and $\mathrm{F}$-actin formation during sperm capacitation. Developmental Biology 267 230-241.

Flaherty SP 1987 Further ultrastructural observations on the sperm head of the plains mouse. Pseudomys australis (Rodentia: Muridae). Anatomical Record 217 240-249.

Flaherty SP, Winfrey VP \& Olson GE 1988 Localization of actin in human, bull, rabbit, and hamster sperm by immunoelectron microscopy. Anatomical Record 221 599-610.

Fouquet JP \& Kann ML 1992 Species-specific localization of actin in mammalian spermatozoa: fact or artifact? Microscopic Research Technology 20 251-258.

Garbi M, Rubinstein S, Lax Y \& Breitbart H 2000 Activation of protein kinase $\mathrm{C} \alpha$ in the lysophosphatidic acid-induced bovine sperm acrosome reaction and phospholipase D1 regulation. Biology of Reproduction 63 1271-1277.

Heid HW, Figge U, Winter S, Kuhn R, Zimbelmann R \& Franke WW 2002 Novel actin-related proteins Arp-T1 and Arp-T2 as components of the cytoskeletal calyx of the mammalian sperm head. Experimental Cell Research 279 177-187.

de las Heras MA, Valcarcel A, Perez LJ \& Moses DF 1997 Actin localization in ram spermatozoa: effect of freezing/thawing, capacitation and calcium ionophore-induced acrosomal exocytosis. Tissue Cell 29 47-53.

Howes EA, Hurst SM \& Jones R 2001 Actin and actin-binding proteins in bovine spermatozoa: potential role in membrane remodeling and intracellular signaling during epididymal maturation and the acrosome reaction. Journal of Andrology 22 62-72.

Kumakiri J, Oda S, Kinoshita K \& Miyazaki S 2003 Involvement of Rho family $G$ protein in the cell signaling for sperm incorporation during fertilization of mouse eggs: inhibition by Clostridium difficile toxin B. Developmental Biology $260522-535$.

Lax Y, Rubinstein S \& Breitbart H 1994 Epidermal growth factor induces acrosomal exocytosis in bovine sperm. FEBS Letters 339 234-238.

Leclerc P, de Lamirande E \& Gagnon C 1997 Regulation of proteintyrosine phosphorylation and human sperm capacitation by reactive oxygen derivatives. Free Radical in Biology and Medicine 22 643-656.

Lefievre L, Jha KN, de Lamirande E, Visconti PE \& Gagnon C 2002 Activation of protein kinase A during human sperm capacitation and acrosome reaction. Journal of Andrology 23 709-716.

Lin M, Hess R \& Aitken JR 2002 Induction of sperm maturation in vitro in epididymal cell cultures of the tammar wallaby (Macropus eugenii): disruption of motility initiation and sperm morphogenesis by inhibition of actin polymerization. Reproduction $\mathbf{1 2 4}$ 107-117.

Liu DY, Martic M, Clarke GN, Dunlop ME \& Baker HW 1999 An important role of actin polymerization in the human zona pellucida-induced acrosome reaction. Molecular Human Reproduction 5 941-949.

Liu DY, Martic M, Clarke GN, Grkovic I, Garrett C, Dunlop ME \& Baker HW 2002 An anti-actin monoclonal antibody inhibits the zona pellucida-induced acrosome reaction and hyperactivated motility of human sperm. Molecular Human Reproduction 8 $37-47$.

Moreno-Fierros L, Hernandez EO, Salgado ZO \& Mujica A 1992 Factin in guinea pig spermatozoa: its role in calmodulin translocation during acrosome reaction. Molecular Reproduction and Development 33 172-181.

Ochs D \& Wolf DP 1985 Actin in ejaculated human sperm cells. Biology of Reproduction 33 1223-1226.

Olson GE \& Winfrey VP 1991 Changes in actin distribution during sperm development in the opossum, Monodelphis domestica. Anatomical Record $230209-217$.

O'Toole CM, Arnoult C, Darszon A, Steinhardt RA \& Florman HM $2000 \mathrm{Ca}^{2+}$ entry through store-operated channels in mouse sperm is initiated by egg ZP3 and drives the acrosome reaction. Molecular Biology of the Cell 11 1571-1584.

Pelletier R, Trifaro JM, Carbajal ME, Okawara Y \& Vitale ML 1999 Calcium-dependent actin filament-severing protein scinderin levels and localization in bovine testis, epididymis and spermatozoa. Biology of Reproduction 60 1128-1136.

Rivlin J, Mendel J, Rubinstein S, Etkovitz N \& Breitbart H 2004 Role of hydrogen peroxide in sperm capacitation and acrosome reaction. Biology of Reproduction 70 518-522.

Rogers BJ, Bastias C, Coulson RL \& Russell LD 1989 Cytochalasin D inhibits penetration of hamster eggs by guinea pig and human spermatozoa. Journal of Andrology 10 275-282.

Sanchez-Gutierrez M, Contreras RG \& Mujica A 2002 Cytochalasin$\mathrm{D}$ retards sperm incorporation deep into the egg cytoplasm but not membrane fusion with the egg plasma membrane. Molecular Reproduction and Development 63 518-528. 
Spungin B \& Breitbart H 1996 Calcium mobilization and influx during sperm exocytosis. Journal of Cell Science 109 1947-1955.

Spungin B, Margalit I \& Breitbart H 1995 Sperm exocytosis reconstructed in a cell-free system. Evidence for the involvement of phospholipase $\mathrm{C}$ and actin filaments in membrane fusion. Journal of Cell Science 108 2525-2535.

Talbot P \& Kleve MG 1978 Hamster sperm cross react with antiactin. Journal of Experimental Zoology 204 131-136.

Tanaka H, Yoshimura Y, Nishina Y, Nozaki M, Nojima H \& Nishimune Y 1994 Isolation and characterization of cDNA clones specifically expressed in testicular germ cells. FEBS Letters 355 4-10.

Tomes CN, McMaster CR \& Saling PM 1996 Activation of mouse sperm phosphatidylinositol-4,5 bisphosphate-phospholipase $C$ by zona pellucida is modulated by tyrosine phosphorylation. Molecular Reproduction and Development 43 196-204.

Virtanen I, Badley RA, Paasivuo R \& Lehto VP 1984 Distinct cytoskeletal domains revealed in sperm cells. Journal of Cell Biology 99 1083-1091.

Visconti PE, Moore GD, Bailey JL, Laclerc P, Connors SA, Pan D, Olds-Clarke P \& Kopf GS 1995 Capacitation in mouse spermatozoa II. Protein tyrosine phosphorylation and capacitation are regulated by a cAMP-dependent pathway. Development 121 1139-1150.
Vogl AW 1989 Distribution and function of organized concentrations of actin filaments in mammalian spermatogenic cells and Sertoli cells. Int Rev Cytol 119 1-56.

Vogl AW, Genereux K \& Pfeiffer DC 1993 Filamentous actin detected in rat spermatozoa. Tissue Cell 25 33-48.

Wassarman PM 1988 Zona pellucida glycoproteins. Annual Reviews in Biochemistry 57 415-442.

Yagi A \& Paranko J 1995 Actin, alpha-actinin, and spectrin with specific associations with the postacrosomal and acrosomal domains of bovine spermatozoa. Anatomical Record 241 $77-87$.

Yanagimachi R 1994 Mammalian fertilization. In The Physiology of Reproduction, pp 189-317. Eds E Knobil \& JD Neil. New York: Raven Press.

Received 17 August 2004

First decision 21 October 2004

Revised manuscript received 9 November 2004

Accepted 15 November 2004 